\title{
Enhanced response in Brillouin distributed optical fibre sensors by simultaneous time and frequency pump multiplexing
}

\author{
Marcelo A. Soto*a , Amelia Lavinia Ricchiuti ${ }^{\mathrm{b}}$, Liang Zhang ${ }^{\mathrm{a}, \dagger}$, David Barrera ${ }^{\mathrm{b}}$, Salvador Sales ${ }^{\mathrm{b}}$, \\ Luc Thévenaz ${ }^{\mathrm{a}}$ \\ ${ }^{a}$ EPFL Swiss Federal Institute of Technology, Institute of Electrical Engineering, \\ SCI STI LT, Station 11, CH-1015 Lausanne, Switzerland; \\ ${ }^{\mathrm{b}}$ ITEAM Research Institute, Universidad Politécnica de Valencia, \\ Camino de Vera s/n, 46022 Valencia, Spain; \\ †Permanent address: Department of Physics and Astronomy, State Key Lab of Advanced Optical \\ Communication Systems and Networks, Shanghai Jiao Tong University, Shanghai, 200240, China; \\ *E-mail: marcelo.soto@epfl.ch
}

\begin{abstract}
A technique to enhance the response of Brillouin distributed sensors is proposed and experimentally validated. The method consists in creating a multi-frequency pump pulse interacting with a multi-frequency continuous-wave probe. The power of each pulse at a distinct frequency is lower than the threshold for nonlinear effects, while the sensor response remains given by the total power of all pulses. Distinct frequency pulses are delayed to avoid temporal overlapping and cross-interaction; this requires to smartly reconstruct the traces before photo-detection. The method is validated in a $50 \mathrm{~km}$-long sensor using 3 frequencies, demonstrating a signal-to-noise ratio enhancement of $4.8 \mathrm{~dB}$.
\end{abstract}

Keywords: Optical fibre sensor, stimulated Brillouin scattering, distributed fibre sensor

\section{INTRODUCTION}

The huge development of Brillouin-based distributed optical fibre sensors during the past few years has turned this technology into one of the most interesting solutions to perform distributed strain and temperature monitoring along many tens of kilometres, with metric spatial resolution. One of the schemes that offers the highest sensing performance is called Brillouin optical-time domain analysis (BOTDA) ${ }^{1}$, which is based on the interaction of two optical waves by stimulated Brillouin scattering (SBS). The standard BOTDA scheme uses a high-power pulsed pump and a counterpropagating continuous-wave $(\mathrm{CW})$ probe signal ${ }^{1-3}$. Since both probe and pump powers equally contribute to the response of the sensor along the entire fibre ${ }^{2}$, the ultimate limit in long-range Brillouin sensing is related to the maximum powers that can be launched into the fibre. More specifically, the peak pump power of Brillouin sensors is limited by the threshold of nonlinearities, essentially modulation instability (MI) ${ }^{4}$, limiting its peak pump power to about $100-150 \mathrm{~mW}$ in standard single-mode fibres. As far as the CW probe power is concerned, it is primarily limited by pump depletion, which distorts the measurements ${ }^{5}$, but this effect can be massively alleviated using a probe signal with two spectral sidebands $^{3,5}$. Probe power is ultimately limited to $\sim 5 \mathrm{~mW}$ by the onset of amplified spontaneous Brillouin scattering.

In this paper a method is proposed and experimentally validated, delivering multiplied pump and probe powers into the sensing fibre, without stimulating detrimental nonlinear effects. The method requires the generation of a pump signal distributed over $N$ different spectral components, which are distinctively mutually delayed. A proof-of-concept experiment is implemented based on two arrays of fibre Bragg gratings (FBGs), which deliver suitable delaying and filtering on the optical signals. Experimental results demonstrate the capability of the technique to upscale the sensor response, while detrimental nonlinear effects are not observed in spite of multiplied pump and probe powers.

\section{DESCRIPTION OF THE PROPOSED METHOD}

The technique proposed in this paper consists in creating a multi-frequency pump pulse interacting with a matching multi-frequency $\mathrm{CW}$ probe wave. In contrast to the standard BOTDA scheme, in which a single frequency pump is used (see Fig. 1(a)), pump pulses showing $N$ frequency components are here employed to produce Brillouin amplification along the sensing fibre. The purpose is to spread the power among multiple frequency components to increase the total power launched into the fibre, while keeping the power in individual pulses below the onset of MI. Each of these $N$

23rd International Conference on Optical Fibre Sensors, edited by José Miguel López-Higuera,

Julian Jones, Manuel López-Amo, José Luis Santos, Proc. of SPIE Vol. 9157, 91575T

(C) 2014 SPIE $\cdot$ CCC code: $0277-786 X / 14 / \$ 18 \cdot$ doi: $10.1117 / 12.2059006$ 
pump frequency components interacts only with one pair of probe components symmetrically located around the pump spectral line, as schematically shown in Fig. 1(b). Such an interaction is similar to the one occurring in a standard BOTDA scheme, as depicted in Fig. 1(a), but in this case the use of $N$ frequency components can be interpreted as a spectral parallelisation of the Brillouin interaction occurring along the distributed sensor. Since the sensor response depends linearly on the pump power in the small gain approximation $^{2}$, the $N$ pulses will generate a response equivalent to a single pulse with an $N$-times larger power. However, the compound signals contains several spectral lines that can all potentially interact; thus, the Brillouin interaction within each pump-probe pair has to remain independent from other pair interactions. A smart choice of the frequency separation $\Delta f$ must be made to avoid unwanted Brillouin interactions with other frequency components.

It is worth mentioning that if pump pulses at different frequencies and with high peak power are launched synchronously into the fibre, their nonlinear parametric interaction during propagation will mutually seed unwanted nonlinear processes. They will consequently be observed at a reduced power and will also lead to strong distortion in the traces, impairing significantly the system performance. In order to maximise the pump power and to avoid parametric interaction among pulses, the pump pulses at the $N$ frequencies have to be simply temporally separated. A wavelength-dependent delay is imposed between pulses, which must be similar or longer than the single pulse duration to secure that pulses do not coexist in time and location inside the sensing fibre. This also guarantees high and optimised peak pump power: although each pump pulse is still limited by the onset of $\mathrm{MI}^{4}$, the total pump power supported by the fibre can be multiplied by an integer factor equal to the number $N$ of frequency components. It must be mentioned that the same technique can be used to avoid co-propagating amplified spontaneous Raman scattering ${ }^{4}$ in normal dispersion fibres where $\mathrm{MI}$ is not observed.

Additionally, considering that the Brillouin interaction between each of the $N$ pump-probe pairs remains independent from each other, the total probe power allowed in the fibre can also be safely multiplied by a factor $N$, while each line is still limited at $5 \mathrm{~mW}^{5}$, as in the standard BOTDA scheme. However, it is important to notice that the delay between the $N$ pulses is expected to generate independent and delayed BOTDA traces. The probe signals containing the Brillouin gain information have therefore to be properly time-shifted before photo-detection by applying a delay opposite to the one originally set to the corresponding pump pulse. Actually this temporal realignment of the $N$ BOTDA traces (carried by the probe components) is essential to avoid ruining the spatial resolution of the sensor.

\section{EXPERIMENTAL SETUP}

The proof-of-concept setup used to verify the feasibility of the proposed technique is shown in Fig. 2. This implementation makes use of $N=3$ frequency components, which are generated by modulating the intensity of a $\mathrm{CW}$ laser source using a Mach-Zehnder modulator (MZM) driven by a microwave signal at $17 \mathrm{GHz}$. The DC bias voltage of the modulator has been adjusted so that three frequency components (carrier and sidebands) are obtained with the same amplitude. This multi-frequency signal is then split into two branches to generate pump and probe waves.

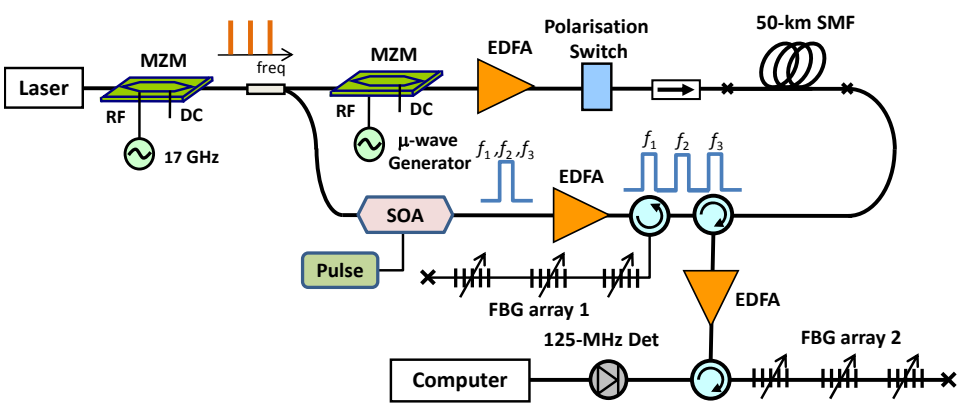

Figure 2. Experimental setup for proposed BOTDA sensor based on multi-frequency de-synchronised pump pulses.

The probe light is created using a MZM operating in carrier-suppression mode, giving rise to two sidebands around each of the $N=3$ original frequencies, so a total of 6 lines. A polarisation switch is used to avoid any gain fading resulting from the polarisation-dependent Brillouin gain. Meanwhile, pump pulses are created by a semiconductor optical amplifier (SOA), in which the gain is gated using a 20 ns-long electrical pulse. Adjusting the output power of erbium-doped fibre amplifiers (EDFAs), probe and pump powers are separately optimised at the fibre input to avoid nonlinear effects.

Using an optical circulator, the pump light is sent into a first array of three $7 \mathrm{~cm}$-long FBGs, having central wavelength at $1551.1 \mathrm{~nm}$ (at room temperature), transmission band of $33 \mathrm{pm}$ full-width at half-maximum (FWHM), high reflectivity (nearly 99\%), and positioned every $5 \mathrm{~m}$ along the fibre. Each FBG has been mounted on a distinct translation stage for a 
selective fine tuning on the $N=3$ distinct frequencies delivered by the first MZM. Thus, by simply adjusting the FBGs, three pulses separated spectrally by $17 \mathrm{GHz}$ and temporally by $50 \mathrm{~ns}$ are obtained.

Pulses and probe components are launched into a $50 \mathrm{~km}$-long standard single-mode fibre (SMF). At the receiver, the multi-frequency probe waves are first amplified by an EDFA, and then sent into a second array of FBGs. These FBGs are properly tuned in order to apply a selective reverse delay to the probe waves, so that the time differences resulting from the delayed pump pulses are compensated. This way the BOTDA traces are automatically properly rearranged to obtain the equivalent trace corresponding to the interaction of the summed pulses. In addition to the delay provided by this second array of FBGs, each FBG also filters out unwanted frequency components as well as the amplified spontaneous emission (ASE) noise originated by the EDFA. The signal is eventually detected by a $125 \mathrm{MHz}$ photodetector, and then acquired with a card connected to a computer.

\section{EXPERIMENTAL RESULTS AND DISCUSSION}

To experimentally validate the proposed method, BOTDA traces at the peak gain frequency have been acquired for both $N=3$ and $N=1$ (standard case). The peak power of the pump pulses has been adjusted to the maximum of $100 \mathrm{~mW}$ per pulse to avoid $\mathrm{MI}^{4}$; so that the total pump power is $300 \mathrm{~mW}$ for $N=3$. The three pulses are properly ordered in time and frequency to avoid nonlinearities, as above described. Fig. 3(a) compares BOTDA traces for both cases, indicating that the use of 3 frequency components increases the sensor response by a factor 3, as a result of the higher total pump power. This corresponds to a signal-to-noise ratio (SNR) enhancement of $4.8 \mathrm{~dB}$. Using the standard scheme $(N=1)$ with a $2 \mathrm{~m}$ spatial resolution and 2000 time-averaged traces, a SNR equal to $6.1 \mathrm{~dB}$ is experimentally observed at the fibre far end. However, using $N=3$ components, the SNR has been improved up to $10.9 \mathrm{~dB}$. Certainly much higher SNR enhancement will be reached if the number of frequency components $N$ is increased.

It is worth noticing that no distortion (potentially originated from nonlinear effects) is observed in the BOTDA trace measured with $N=3$ (red curve), though high pump power $(300 \mathrm{~mW})$ is launched into the fibre. Actually the delay between pulses at different frequencies plays a crucial role in the method: if the three $100 \mathrm{~mW}$ pulses at distinct frequencies are synchronously sent into the fibre, the parametric interaction among the spectral components leads to distorted traces. Fig. 3(b) actually compares BOTDA traces obtained with (red curve) and without (blue curve) delays between pulses. Interestingly, the same sensor response is observed in both cases at the fibre input (due to the same input pump power); however, when no delay is applied, nonlinearities resulting from parametric interaction among pulses produce strong oscillations in the effective pump power, and hence in the measured Brillouin gain. These oscillations are eliminated using the wavelength-dependent delays, leading to a trace (red curve) with no distortion. Pump depletion has been measured to be below $4 \%$, resulting in negligible additional distortion in the traces ${ }^{5}$.
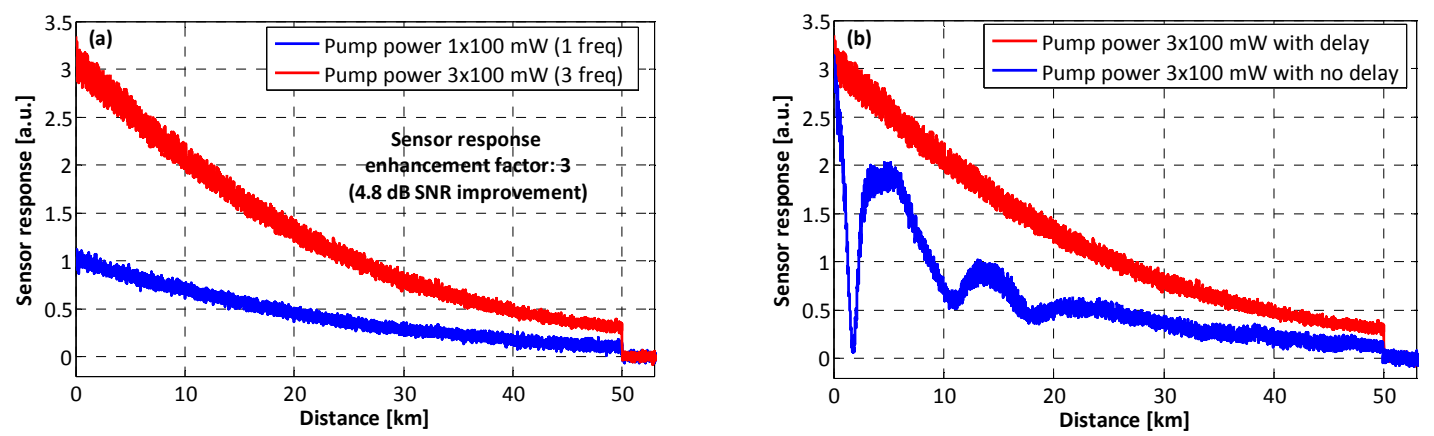

Figure 3. Sensor response measured at the peak gain frequency. (a) Comparison between the standard BOTDA ( $N=1)$ and the proposed scheme using $N=3$ and delayed pump pulses. (b) Traces measured with $N=3$ frequency components, with (red curve) and without (blue curve) delay between pump pulses.

Fig. 4 shows the Brillouin gain spectrum versus distance, measured using $1 \mathrm{MHz}$ frequency step and $N=3$ spectral components. Thus, fitting the spectrum a parabolic curve ${ }^{2}$, the Brillouin frequency profile is obtained, as shown in Fig. 4 inset. Calculating the standard deviation of the measured Brillouin frequency along the fibre, the final uncertainty of the sensor has been estimated for the standard BOTDA configuration $(N=1)$ and for the scheme using $N=3$ spectral components. Fig. 5 shows the frequency uncertainty versus distance for both cases, indicating that the accuracy of $1.8 \mathrm{MHz}$ - obtained with the standard scheme at a distance of $50 \mathrm{~km}$ - can be improved down to $0.6 \mathrm{MHz}$ using the proposed method with $N=3$. This corresponds to a factor 3 in the uncertainty reduction, in perfect agreement with the prediction of a $4.8 \mathrm{~dB}$ SNR enhancement ${ }^{2}$. 
Finally, the spatial resolution of the method has been experimentally verified. For this purpose, $2 \mathrm{~m}$ of the sensing fibre near the farthest end have been heated up to $50^{\circ} \mathrm{C}$, while the rest of the fibre is kept at room temperature $\left(24^{\circ} \mathrm{C}\right)$. This is actually a crucial test for this method, considering the pulses de-synchronisation followed by the re-synchronisation of the traces. Possible imprecisions in the reverse delays delivered by the second FBGs array will certainly jam the spatial information. Fig. 6 shows the hot-spot detection measured near a $50 \mathrm{~km}$ distance using the proposed technique with $N=3$ frequencies. Results indicate a correct determination of the temperature within the hot region and no penalty on the $2.0 \mathrm{~m}$ spatial resolution is observed with the $20 \mathrm{~ns}$ de-synchronised pump pulses.

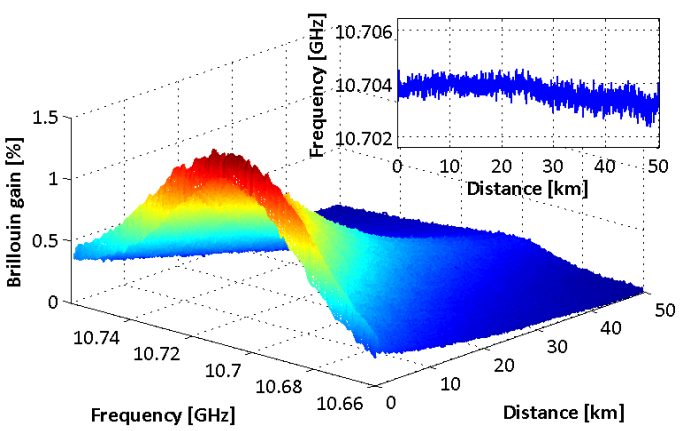

Figure 4. Measured Brillouin gain spectrum versus distance. Inset: Retrieved Brillouin frequency profile.

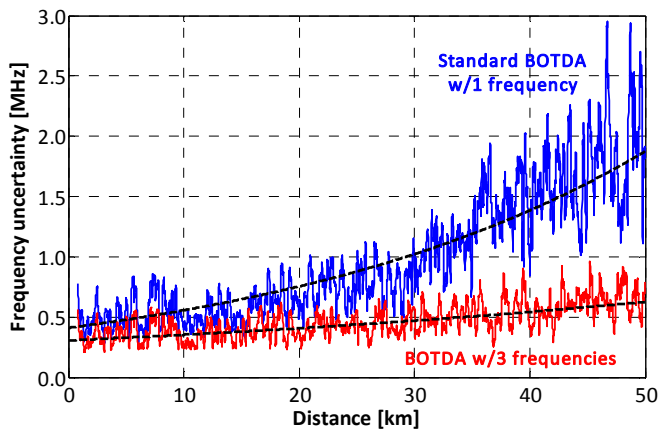

Figure 5. Frequency uncertainty versus distance, for BOTDA schemes using $N=1$ (standard) and $N=3$ frequency components

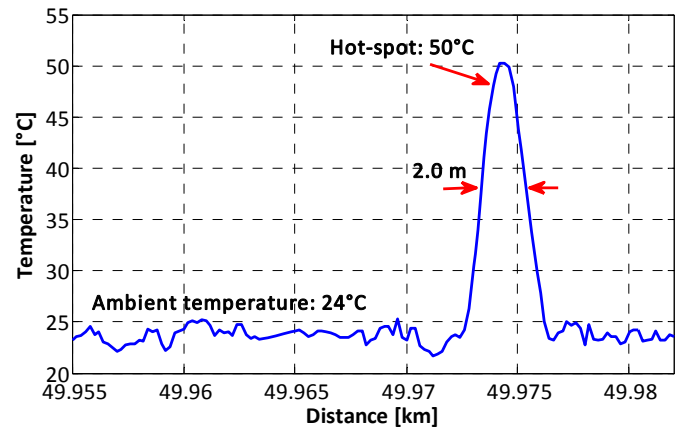

Figure 6. Hot-spot detection at the far fibre end (around $50 \mathrm{~km}$ distance), demonstrating $2 \mathrm{~m}$ spatial resolution

\section{CONCLUSION}

A technique to enhance the response and performance of BOTDA sensors has been proposed and experimentally validated, which is based on an elegant method to by-pass the fundamental limits given by fibre nonlinearities. The response of the sensor can be arbitrarily multiplied by integer steps and is just limited by the added complexity of the passive time-frequency mux-demux unit. The additional SNR provided by the method can be useful to extend the sensing range, to reduce measurement time, and/or to improve spatial and measured resolutions. The technique offers many advantages when compared to alternative approaches: simple direct detection can still be employed, avoiding the use of sophisticated detection schemes based for instance on coherent detection. Furthermore, the frequency scan, acquisition procedure and data processing remain unmodified from the basic BOTDA scheme. The configuration presented here as a proof of concept, based on FBGs arrays, is certainly not the only possible implementation and schemes using more advanced devices with better intrinsic stability are under consideration.

This work was performed in the framework and with the support of the COST Action TD1001 OFSeSa. M. A. Soto and L. Thévenaz acknowledge the support from the Swiss Commission for Technology and Innovation (Project 13122.1). UPVLC group acknowledges the support from the Spanish MICINN and the Valencia Government through the projects TEC201129120-C05-05 and ACOMP/2013/146, respectively. L. Zhang acknowledges the support from the China Scholarship Council.

\section{REFERENCES}

[1] Horiguchi, T., Shimizu, K., Kurashima, T., Tateda, M. and Koyamada, Y., "Development of a distributed sensing technique using Brillouin scattering," J. Lightwave Technol. 13(7), 1296-1302 (1995).

[2] Soto, M. A. and Thévenaz, L., "Modeling and evaluating the performance of Brillouin distributed optical fiber sensors," Opt. Express 21(25), 31347-31366 (2013).

[3] Nikles, M, Thévenaz, L. and Robert, P. A., "Brillouin gain spectrum characterization in single-mode optical fibers," J. Lightwave Technol. 15(10), 1842-1851 (1997).

[4] Foaleng, S. M. and Thévenaz, L., "Impact of Raman scattering and modulation instability on the performances of Brillouin sensors," Proc. SPIE 7753, 77539V (2011).

[5] Thévenaz, L., Foaleng Mafang, S. and Lin, J., "Effect of pulse depletion in a Brillouin optical time-domain analysis system," Opt. Express 21(12), 14017-14035 (2013). 